\title{
RANGE Application in Examination-oriented Education: Focusing on TEM-8 Translations Shang-chun ZENG ${ }^{1}$ and Ying SHEN ${ }^{2, *}$ \\ ${ }^{1}$ College of International Exchange, Wuzhou University, Guangxi, China \\ ${ }^{2}$ Foreign Languages College, Guangxi University, Guangxi, China Institute for Intelligent Systems, the University of Memphis, TN USA
}

${ }^{*}$ Corresponding author: Ying SHEN

Keywords: RANGE, TEM-8, Translation.

\begin{abstract}
As an important part of Test for English Majors Band-8 (TEM-8), an inclusive test to figure out the students' levels of English learning, translation (translation from Chinese to English and fromEnglish to Chinese) is of certain difficulty and accounts for a large percentage of grades. Being a corpus analysis software, RANGE contributes a lot to word output and word acquisition. It carries several authoritative word lists, which are used to compare and analyze the words of the selected texts, so the researchers can get gradable words. This thesis aims to help the students English learners especially English majors further lay the foundation and enlarge their vocabulary to improve translation grades in TEM- 8 by collecting and analyzing original tests and corresponding drills via RANGE, and figure out the types of words that most commonly used and some skills in word usage. Meanwhile, the result can also be applied to other English tests. Compared with conventional methods, specific research with RANGE will improve the students' ability to pass English tests and to develop their learning efficiency.
\end{abstract}

\section{Introduction}

Translation, as a dispensable means by which different individuals and peoples from different ethnic groups and countries communicate and exchange ideas with each other, and as a tie that bridges and motivates human civilizations as well as their development and heritage, is an important part of our language activities. As to an English major, his/her comprehensive skills on translation are intensively reflected in the transformation between the two languages. The materials of translation part of TEM-8 cover a wide range of topics, including literature, humanity, culture, speeches of famous figures, linguistics and so on. Unlike single sentence translation, the translations of TEM- 8 calls for not only such basic knowledge as vocabulary and sentence structure, but also the ability to understand the context and conduct logical deduction so as to make the translation match with the whole passage.

RANGE is a corpus analysis software designed by two linguists from Victoria University of New Zealand, Professor P . Nation and A. Coxhead, and programmed by A. Heatley. It is designed to analyze the extent and profundity of the words in a passage. Word frequency as the basic design, RANGE carries several basic vocabulary lists that cover different levels of words in English. By comparing the words of certain materials with the vocabulary lists, researchers can thus work out the results that show the word distribution status of various types of articles and passages. It can also show the frequency of the word family and the tokens as well as the sum of their accumulated frequency within the materials. What is more, 
RANGE can also compare and contrast the vocabulary and the use of rhetoric of two different passages.

So far, a series of studies have been made by researchers both home and abroad. Foreign researchers have analyzed and deducted the common words used in different passages, make predictions on the vocabulary needed when readers are reading a certain type of writing, and test the vocabulary of those whose mother tongue is not English (Cobb \& Horst 2001). In recent years, researchers of our country are paying more attention to RANGE.

\section{Research Procedures}

\section{Research Method}

The objects of my study are translations of TEM-8, including translating from Chinese into English and from English into Chinese. The materials examined are original tests from TEM- 8 from 2005-2015, simulation questions and samples of some randomly chosen students' work, as the first and second sources of research objects respectively.

The research is conducted by means of quantification, simplification and optimization. Firstly, as many as materials regarding TEM-8 translation should be collected, including class drills, simulation exercises and original texts in order to ensure diversity and authority. Secondly, all materials are systematically sorted out, filtering those that are familiar and identical to make sure that the output data are more convincing. Thirdly, those materials will be refined and re-matched according to their types and linguistic features. Throughout this process the materials are now authentic and organically organized so that this research results are both comprehensible and targeted.

\section{Research Operation}

RANGE is loaded with three major authoritative vocabulary lists, and they are BASEWORD1, BASEWORD2, and BASEWORD3. There are 1000 word families (999 indeed) that are commonly used in BASEWRD1, 1000 word families (987 indeed) are less commonly used, and 570 word families are academic words which are commonly used in junior and higher education. Tokens are used to describe the total times a word appear in a certain piece of material and its corresponding percentage. Types indicate how many times a word is initially used as a single word in a certain piece of material and its corresponding percentage. As to those words that are not in the three lists, their word families will be "not in the list".

Before running the program to process the materials, I have to make sure that the file is in ".txt" format. The operation processes of the software are the following: firstly, click on the icon named "Range 32" to start the program; secondly, click on "File" and find the targeted file, and then select it; thirdly, click on "File" again and click on "Save"; lastly, click on "Process files" to run the program and the results will be shown.

\section{Results and Discussions}

\section{General Analysis of TEM-8 Translation}

The following data are part of the the results of the materials of original tests from 2005 to 2015 and simulation questions. We can see from the tokens and types that there are in total 17794 words, and there are 4091 words if each word is calculated once only. Among those 
words there are 1686 found in BASEWORD 1, accounting for about $41.21 \%$ in terms of all the lists, and the tokens are 14189 , accounting for about $79.74 \%$ of the total tokens; there are 589 words found in BASEWORD 2, accounting for about $14.62 \%$ of all the lists, and the tokens are 974 , accounting for about $5.47 \%$ of the total tokens; there are 434 words found in BASEWORD 3, the proportion of which is $10.61 \%$, and the tokens are 716 , the proportion of which is $4.02 \%$. Apart from them, there are 1373 words not in the list, accounting for about $33.56 \%$ and the tokens are 1915 , accounting for approximately $10.76 \%$. Compared to the listening and writing part, the words' tokens and types which do not belong to the three lists account for larger percentages, which means it is more common to encounter words that are difficult to understand, and that pushes the students to expand their vocabulary.

Li (2007) and Zhou et al. (2008) have contributed a lot to the study of Chinese students' vocabulary. According to their studies, a lack of called-for vocabulary and misspellings are often seen in the examinations. Therefore, RANGE can lead the students to purposefully know exactly what words they need to be loaded with under different types of writing and contexts, so that they can develop a quick-response word tank in their mind where words show various genres, thus the faithfulness and accuracy of the translations can be highly improved. It is also recommended that the students should do as many exercises as they can to ensure the words they pick up from the results can be truly mastered. Based on the data results, functional words such as definite and indefinite articles and prepositions and substantive words such as link verbs and pronouns are the most commonly seen among all of the words. It is also noted that grammatical adverbs and modal verbs are important parts of the word formation.

\section{Functional Words in TEM-8 Translation}

\section{Prepositions}

According to the results via Frequency of tests from 2005 to 2015 and simulation drills, among the 100 words that are of the highest usage frequency, there are 17 prepositions, covering nearly all of the commonly used ones in English here are the results.

Based on the data it can be concluded that firstly functional words are of huge significance and they should be used properly under any circumstance. Subtle as it appears to be, the functional word plays an important role in linking sentences and forming contexts as the joints of a translation, and a translation of high quality lies in the proper use of functional words because English is a logical language while Chinese stresses on conveying meaning. This is especially noticeable when translating a piece of material from Chinese to English. We have to figure out the internal logical structure of the Chinese and then restructure the words and sentences to match the English expression by adding appropriate functional words; otherwise misunderstanding or even mistaken meanings may be delivered.

Analysis on the materials shows that prepositions are often used with nouns, verbs and adjectives to form phrases and phrasal verbs. A lot of prepositions have their fixed matches according to the quantity, the nature, time and locations of the modified objects. From this point of view, students ought to pay attention to different prepositional matches. The following are some commonly used prepositional phrases in TEM-8 translation, and the corresponding prepositions are statistically of high frequency:

stick to, refer to, turn to, occur to, attend to, lead to, happen to, get to, hold on to, belong to, adapt to, attribute to, object to/against, subscribe to, accede, yield, resort to, succumb to, married to, dedicated to, parallel to, relevant to, indifferent to, devoted to/into, contrary to, 
inferior to, superior to, proportionate to, accessory to, accustomed to, adjacent to, akin to, adhere to, congenial to, obedient to, peculiar to, preferable to, prior to, prone to, subsequent to, susceptible to, consist of, die of, rob of, accuse of, cheat of, convince of, relieve of, deprive of, derive of, act on, call on/off, insist on, count on, reckon on, fix on, escape from, depart from, descend from, refrain from, expel from, abstain from, distinguish from

\section{Pronouns}

According to the results via Frequency of tests from 2005 to 2015 and simulation drills, among the 100 words that are of the highest usage frequency, there are 33 pronouns, accounting for about a third of it. Ranking in reverse order, the most commonly used pronouns are that, it, $I$, we, they, their, one, all, this, which, my, there, when, she, what, our, he, more, his, you, its, her, other, who, those, us, them, some, any, another, your me and as, some of which are also grammatical adverbs. Basically, these pronouns cover all of the types, including directive, personal, questionable, relative and possessive ones. This shows that a wide use of pronouns is one of the major features in English, which can simplify the expression. However, sometimes it may also cause obscure and misunderstanding, and that needs the students to deeply analyze the sentence structures and understand the tests through contexts.

\section{Relative Adverbs}

Moreover, due to the fact that there are structure and pattern differences between the language of English and Chinese, the application of relative adverbs will make it clear that the actual order and cause and effect can be expressed clearly. In English there are multiple compound sentences, which calls for various relative adverbs. When doing translation from Chinese to English, combining several sentences via the relative adverbs is a good way to make the translation more authentic and enrich sentence patterns. Apparently, this is based on the students' solid knowledge foundation and clear thoughts and logic in order to avoid ambiguity or tangles.

It can be concluded from the 100 words that are most commonly used in TEM- 8 translation that majority of them are pronouns, prepositions, conjunctions or relative adverbs. It is noted that a certain word may be used with different meaning. For instance, in the texts "In the late $19^{\text {th }}$ century, there was a tendency to think of each of the arts...in general, musicians in Russia...of the musical compositions...", prepositions of and in respectively have different meanings, and in some cases of can be replaced by among. This tells the students that word diversity should be considered an important means to enhance the readability of the translations.

Let us look at the following passage through which we can learn that several functional words such as cause and result conjunctions have been used to make the translation smooth and logically understandable.

Chinese people has never thought of human being as the highest creature among everything since ancient times, whose reflection takes a quite appropriate proportion with all others in our natural world in both aspects of philosophy and arts, but not as an absolute dominant ruler. Therefore, our bitterness and depression are basically less than those of westerners, because the intensity of which is growing with the expansion of one's desire and ambition. People in the agriculture society enjoyed far less than people in the industry society, thus their wants are far less either. Besides, ancient Chinese always regard "not confined by material, not driven by material" as the major philosophy. (TEM-8. 2006) 


\section{Modal Verbs}

Can, should and must are of different degrees of emotions, raging from low to high. Generally speaking, can indicates permission and possibility, and it is the most commonly used one in spoken or written English. Should indicates a sense of suggestion, and somehow it sounds more stricter than can. Must indicates giving order or alarming, and it is usually used when the climate of the writing is strict and solemn. Given that the materials of the translation part are mostly lyrical judgmental, critical or argumentative, the use of modal verbs should be put in an important position and especially in prose where the authors' feelings and attitudes as wells as the hidden meanings of a certain passage can be expressed by modal verbs and thus trivial emotions can be sensed through these verbs. Using modal verbs properly will help polish the translations.

\section{Translation Comparison}

Table 1: Statistics of Sample One

\begin{tabular}{|l|l|l|l|}
\hline WORDLIST & TOKENS/\% & TYPES/\% & FAMILIES \\
\hline one & $105 / 81.40$ & $61 / 72.62$ & 56 \\
\hline two & $14 / 5.41$ & $12 / 8.82$ & 11 \\
\hline three & $12 / 4.63$ & $11 / 8.09$ & 11 \\
\hline Not in the lists & $16 / 6.18$ & $15 / 11.03$ & $? ? ? ? ?$ \\
\hline Total & 259 & 136 & 108 \\
\hline
\end{tabular}

Table 2: Statistics of Sample Two

\begin{tabular}{|l|l|l|l|}
\hline WORDLIST & TOKENS/\% & TYPES/\% & FAMILIES \\
\hline one & $217 / 83.78$ & $98 / 72.06$ & 86 \\
\hline two & $4 / 3.10$ & $4 / 4.76$ & 4 \\
\hline three & $7 / 5.43$ & $7 / 8.33$ & 7 \\
\hline Not in the lists & $13 / 10.08$ & $12 / 14.29$ & $? ? ? ? ?$ \\
\hline Total & 129 & 84 & 67 \\
\hline
\end{tabular}

The above two lists are the results of TEM-8 translation from Chinese to English in 2010, one being the reference answer, the other being the answer of an English major. We can clearly see from the data that generally speaking the words of reference answer are of higher level that those of the student's answer. The words of the student's answer mostly belong to BASEWORD1, while the reference answer uses more relatively advanced words. This means the translation of the reference answer is more accurate and precise. On the contrary, the student's answer may also be acceptable and can convey the meaning just as fine as the reference answer, but it will suffer a loss of marks in terms of word authenticity and diversity in expression.

\section{Conclusions}

As analyzed above, RANGE plays an significant role in assisting English majors to enlarge their vocabulary and enhance accuracy of the word usage, thus makes their translations meet the standards of TEM-8. So far, the research results intensely reflect that prepositions, pronouns, relative adverbs and modal verbs are abundantly used. Students should pay more attention to the use of functional words. Meanwhile, words of WORDBASE3 and words not in the lists are also worth being focused on because they are the keys to make the translations stand out. 
Research studies from both home and abroad have shown that RANGE as a corpus tool can relatively objectively reflect the disparities on lexical richness between two certain texts. In general, RANGE can be applied to assess the students' translations in terms of word accuracy and richness, strengthen their understanding of translation theories and skills by integrating them into word usage, and thus make the translations more authentic and readable. Reversely, the research on TEM-8 translations with the help of RANGE can further exploit the potential of RANGE and find other fields where it can also be applied. For instance, we may analyze TEM-8 reading according to different topics and genres it covers, or uses RANGE as a translation corpus in English and Chinese mutual translation courses.

Admittedly, this thesis only covers a part of the function of software and there is still more to discover. RANGE can be applied to other English tests such as IELTS, TOFEL, BEC, and GMAT. These tests are more specific and knowledge-oriented. Words that have similar meanings or the meanings that are related may appear in the same contexts, and they form a lexical bond. The lexical bond can be further developed with the help of RANGE, which will help the students build their word packages and memorize words. When taking examinations or doing translation of a certain writing style, the students can narrow down the scope and rapidly figure out which word to use so as to better finish their works and save time.

\section{Acknowledgment}

This research is financially supported by the following research foundations:

1. Innovative Research Foundation for National University Students in 2014 (Grant NO.141059349).

2. Distinguished University Teachers' Overseas Detachment Foundation in 2014-2015 from Guangxi Higher Education Bureau.

\section{References}

[1] P. Nation \& A. Coxhead. RANGE. <http: / /www. vuw. ac. nz/ lals/ staff /Paul Nation, 2001.

[2] S. B. Zhou, Y. Gu \& L.Y. Guo. Evaluation of vocabulary size, Foreign Language Field 2008 (1), pp. 9-14.

[3] T. Cobb \& M. Horst. Growing academic vocabulary with a collaborative online database. In B. Morrison, D. Gardner, K. Koebke, \& M., Spratt (Eds.), LT Perspectives on IT \& Multimedia. Hong Kong: Polytechnic University Press, 2001, pp. 189-226.

[4] X. Li. Correlation between vocabulary size, vocabulary depth, and language comprehensive ability, Foreign Languages Teaching and Research 2007 (9), pp. 353-358. 